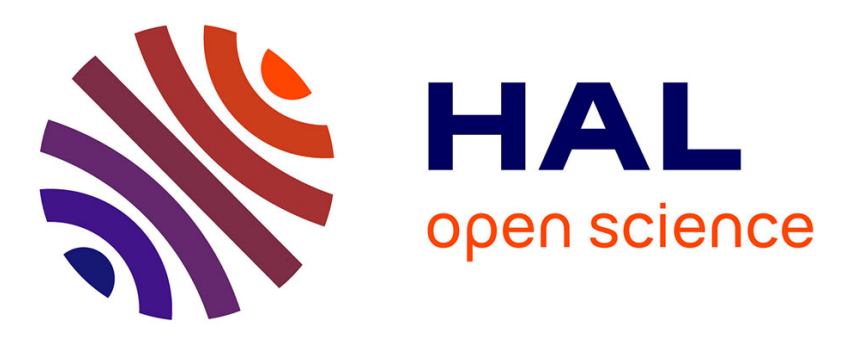

\title{
Estimation of the battery state of charge: a switching Markov state-space model
}

Jana Kalawoun, Patrick Pamphile, Gilles Celeux, Krystyna Biletska, Maxime

Montaru

\section{- To cite this version:}

Jana Kalawoun, Patrick Pamphile, Gilles Celeux, Krystyna Biletska, Maxime Montaru. Estimation of the battery state of charge: a switching Markov state-space model. EUSIPCO'2015, Aug 2015, Nice, France. pp.5. hal-01168344

\section{HAL Id: hal-01168344 \\ https://hal.archives-ouvertes.fr/hal-01168344}

Submitted on 25 Jun 2015

HAL is a multi-disciplinary open access archive for the deposit and dissemination of scientific research documents, whether they are published or not. The documents may come from teaching and research institutions in France or abroad, or from public or private research centers.
L'archive ouverte pluridisciplinaire HAL, est destinée au dépôt et à la diffusion de documents scientifiques de niveau recherche, publiés ou non, émanant des établissements d'enseignement et de recherche français ou étrangers, des laboratoires publics ou privés. 


\title{
ESTIMATION OF THE BATTERY STATE OF CHARGE: A SWITCHING MARKOV STATE-SPACE MODEL
}

\author{
Jana Kalawoun $^{\star} \quad$ Patrick Pamphile Gilles Celeux $^{\dagger} \quad$ Krystyna Biletska ${ }^{\star}$ Maxime Montaru* \\ * CEA, LIST, Laboratoire d'Analyse de Données et Intelligence des Systèmes, 91191 Gif-sur-Yvette, France \\ † INRIA Université Paris Sud CNRS UMR8628 91405 Orsay, France \\ * CEA, LITEN, Laboratoire du Stockage ElectroChimique, 73375 Le Bourget-du-Lac, France
}

\begin{abstract}
An efficient estimation of the State of Charge $(\mathrm{SoC})$ of a battery is a challenging issue in the electric vehicle domain. The battery behavior depends on its chemistry and uncontrolled usage conditions, making it very difficult to estimate the $S o C$. This paper introduces a new model for $S o C$ estimation given instantaneous measurements of current and voltage using a Switching Markov State-Space Model. The unknown parameters of the model are batch learned using a Monte Carlo approximation of the EM algorithm. Validation of the proposed approach on an electric vehicle real data is encouraging and shows the ability of this new model to accurately estimate the $S o C$ for different usage conditions.
\end{abstract}

Index Terms - State of Charge, Kalman Filter, Switching Markov State-Space Model, EM algorithm, Particle Filter

\section{INTRODUCTION}

Nowadays, to achieve better fuel efficiency and reduce toxic emissions, more and more vehicles are powered with an electric motor. Similarly to the fuel gauge in an internal combustion engine vehicle, the State of Charge $(S o C)$ of the battery in an electric vehicle indicates its available energy. Besides, beyond the framework of the automobile industry, the $S o C$ estimation helps prevent overcharge and deep discharge of the battery, which may cause a permanent damage.

A battery being a complex electrochemical system, there is no sensor to measure its $S o C$. Embedded applications, like electric vehicles, impose hardware and time constraints. Therefore, the $S o C$ must be accurately online estimated. A review of methods and models used for $S o C$ estimation as well as their performances in embedded applications is given in [1]. The two most common approaches for $S o C$ estimation are founded on the "Coulomb counting" model and on a general state-space model. The Coulomb counting models the $S o C$ by a weighted summation of the input and output battery

The authors would like to thank Michelin for integrating the CEA pack on a Michelin vehicle and for performing tests on the Michelin Ladoux circuit. This work was supported by the French agency ADEME, as part of the FOREWHEEL project. currents. Despite its simplicity, this method is an open loop and the error of the current sensor can drift the estimation. As a result, this method requires an accurate, thus expensive, current sensor. A general state-space model, combines the $S o C$ modeled by Coulomb counting and the voltage modeled by an equivalent electric circuit. Thus a recursive $S o C$ estimation can be provided by an extended Kalman filter. The key advantage of a Kalman filter is that it is a closed loop method which can take the sensor error into account. However on a real-life electric vehicle, the state-space model describing the battery behavior should change over time. Indeed, these changes are random since they depend not only on uncontrolled external conditions, like ambient temperature and current profile, but also on internal conditions like internal resistance and battery aging. Improvements of the Kalman filter method to include the possibility of changes over time have been approached by identification of the parameters for several temperatures and $S o C \mathrm{~s}$ as in [2], or by including the set of parameters in the state vector as in [3]. These solutions remain limited as in the former the parameters changes according to an estimated, thus may inaccurate, $S o C$ and the latter requires high computational capacity and thereby not suitable for an online application. Changes can also be modeled through a regression function relating each parameter to a given temperature as in [4]. This method omits the influence of the other uncontrolled internal and external conditions. Up to our best knowledge, there is no model or method that gives a reliable online $S o C$ estimation regardless of internal and external conditions.

This paper introduces a new model for the $S o C$ estimation using a Switching Markov State-Space Model (SMSSM): the battery behavior is described by a set of potential linear statespace models, switching randomly according to a Markov chain. The model includes two latent variables: a continuous one, the $S o C$ and a discrete one, the finite Markov state. Two issues arise with this modeling. The first one relates to the inference of unknown parameters. For this purpose, a Monte Carlo approximation of the EM algorithm is used. The second one relates to the choice of the number of hidden Markov states. Being a result of a compromise between accuracy requirements and model complexity, the optimal number of hidden Markov states is assessed using different model selection 
criteria. Numerical experiments were made with electric vehicle real data for different drives and ambient temperatures, and show the potential benefits and the practical usefulness of the proposed model.

The paper is organized as follows. The $S o C$ model is described in Section 2. The parameters estimation is described in Section 3 and discussed in Section 4 using real-life electric vehicle data. Section 5 concludes the paper.

\section{STATE OF CHARGE MODEL}

The battery behavior is observed on $[0 ; T]$, at sampling time points $t$ with a step $\Delta t$ : the current is considered as an input, the voltage is measured, and the $S o C$ is unobserved.

\subsection{Coulomb counting}

The leading $S o C$ estimator is the Coulomb counting:

$$
S o C_{t}=S o C_{0}+\int_{0}^{t} \frac{\eta \cdot I_{s}}{C_{r e f}} d s,
$$

where $\eta$ is the Faraday efficiency, $C_{\text {ref }}$ the reference capacity and $I_{t}$ the algebraic current measurement: positive for a charge and negative for a discharge. This method suffers from error accumulation over time that may introduce bias to the estimated $S o C$. To improve it, the voltage of the battery is generally considered. Indeed, an accurate voltage sensor is not costly contrary to a current sensor. Thus the voltage model depending on the $\mathrm{SoC}$ completes the Coulomb counting in order to establish the so-called linear state-space model.

\subsection{Linear State-Space Model}

Let $X_{t}$ denote the $S o C$ at time $t$ and $Y_{t}$ the voltage. In this paper, we use the standard convention whereby capital letters denote random variables, whereas lower letters are used for their corresponding realizations. To describe the relation between the voltage and the $S o C$, an equivalent circuit of the battery is used. This circuit implements a voltage source representing the open circuit voltage of the battery, and an ohmic resistance describing the internal resistance. The voltage $Y_{t}$ is then given by the "observation equation":

$$
Y_{t}=C \cdot X_{t}+D_{1} \cdot u_{t}+D_{2}+\varepsilon_{t},
$$

where $C, D_{1}$ and $D_{2}$ are constant with physical interpretation and $\varepsilon_{t} \sim \mathcal{N}\left(0, \sigma_{Y}^{2}\right)$ models the error of the voltage sensor. The description of the model is completed by the "transition equation" which is based on Coulomb counting:

$$
X_{t}=X_{t-1}+B u_{t}+\omega_{t},
$$

with $B=\frac{\eta}{C_{r e f}}, u_{t}=I_{t} \Delta t$ and $\omega_{t} \sim \mathcal{N}\left(0, \sigma_{X}^{2}\right)$ modeling the random fluctuations of the $S o C$. The Gaussian white noises $w_{t}$ and $\varepsilon_{t}$ are assumed to be independent. In practice at time $t=0$, the battery is often in a resting state, thus $S o C_{0}$ can be

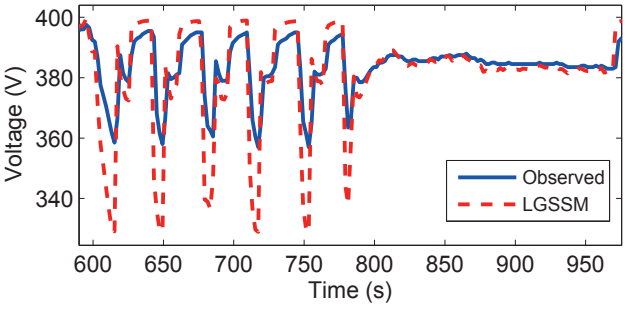

Fig. 1. Prediction of the voltage using a LSSM

efficiently calculated through the open circuit voltage measurement [5]. Thus, the Linear State-Space Model (LSSM) relates the unobserved $X_{t}$ and the observed $Y_{t}$ through linear equations (2) and (3). The Kalman filter provides an optimal estimation of $x_{t}$, in a mean square error sense, given an observation $y_{1: t}=\left\{y_{1}, \ldots, y_{t}\right\}$ and an input $u_{1: t}$ sequences [6].

In practice the battery dynamics change during charge/ discharge according to uncontrolled internal and external conditions. Consequently, each set of usage conditions should be described by specific equations of observation and transition. Let us consider a simple case for which the current and the temperature are constant. In order to monitor the relevance of the Kalman filter, attention has been given to the comparison between the observed and estimated voltage. Figure 1 shows that a single LSSM cannot estimate accurately the voltage throughout the whole interval $[0 ; T]$. Therefore, we suppose that $\left\{X_{t}, Y_{t}\right\}$ is described by different potential LSSMs, and that the changes are random according to an unobserved Markov chain. Hence, the $S o C$ is modeled by the so-called Switching Markov State-Space Model (SMSSM) [7].

\subsection{Switching Markov State-Space Model}

Let us denote $S_{t}$ the indicator of the random switch of LSSM. $S_{t}$ is a Markov chain on $\{1, \ldots, \kappa\}, \Pi(i)=p\left(S_{0}=i\right)$ is its initial distribution and $A(i, j)=p\left(S_{t+1}=j \mid S_{t}=i\right)$ its transition matrix. The switching times are unknown, thus $A$ and $\kappa$ need to be estimated. The $S o C$ is modeled by the following SMSSM:

$$
\begin{aligned}
X_{t} & =X_{t-1}+B\left(S_{t}\right) \cdot u_{t}+\omega_{t} \\
Y_{t} & =C\left(S_{t}\right) \cdot X_{t}+D_{1}\left(S_{t}\right) \cdot u_{t}+D_{2}\left(S_{t}\right)+\varepsilon_{t},
\end{aligned}
$$

where $\omega_{t} \sim \mathcal{N}\left(0 ; \sigma_{X}^{2}\left(S_{t}\right)\right)$ and $\varepsilon_{t} \sim \mathcal{N}\left(0 ; \sigma_{Y}^{2}\left(S_{t}\right)\right)$. The observations $y_{1: T}$ are assumed conditionally independent given $\left(x_{t}, s_{t}\right)$; while $\left\{X_{t}, S_{t}\right\}$ is a Markov chain, verifying

$$
p_{\theta}\left(s_{t}, x_{t} \mid s_{t-1}, x_{t-1}\right)=p_{\theta}\left(s_{t} \mid s_{t-1}\right) p_{\theta}\left(x_{t} \mid x_{t-1}, s_{t}\right),
$$

where $\theta$ is the vector of parameters:

$$
\theta=\left\{B(s), C(s), D_{1}(s), D_{2}(s), \sigma_{X}(s), \sigma_{Y}(s), A\right\}_{1 \leq s \leq \kappa} .
$$

The distributions $p_{\theta}\left(x_{t} \mid x_{t-1}, s_{t}\right)$ and $p_{\theta}\left(y_{t} \mid x_{t}, s_{t}\right)$ are assumed to be Gaussian, whose parameters are deduced from 
(4) and (5). In the case of a specific Markov state sequence $s_{0: T}$, the Kalman filter provides an optimal estimation of $x_{0: T}$, given observation $y_{1: T}$ and input $u_{1: T}$ sequences; otherwise $s_{0: T}$ should be estimated. This point is discussed in $\S 4.3$. In the next section, the problem of estimating the unknown parameters $\theta$ for a fixed $\kappa$ is treated.

\section{BATCH LEARNING OF PARAMETERS}

Let us consider a learning dataset $\left\{y_{1: T}, u_{1: T}\right\}$ where $y_{1: T}$ is observed and $u_{1: T}$ is an input. Here both $x_{0: T}$ and $s_{0: T}$ are unknown. In the following, a batch learning of unknown parameter $\theta$ using the Maximum Likelihood (ML) inference is proposed. The original ML estimation problem can be formulated as follows

$$
\hat{\theta}=\arg \max _{\theta} p_{\theta}\left(y_{1: T}\right)
$$

For SMSSM, the marginal likelihood $p_{\theta}\left(y_{1: T}\right)$ is given by:

$$
p_{\theta}\left(y_{1: T}\right)=\sum_{s_{0: T}} \int_{x_{0: T}} p_{\theta}\left(x_{0: T}, s_{0: T}, y_{1: T}\right) d x_{0: T},
$$

where $p_{\theta}\left(x_{0: T}, s_{0: T}, y_{1: T}\right)$ is the complete-likelihood. It is clear that a direct evaluation of (8) is analytically difficult. Therefore, $\theta$ is estimated with the EM algorithm which is the most widely used method for ML estimates of unknown parameters in models involving latent variables [8].

\subsection{EM algorithm}

The EM algorithm consists of iteratively estimating the set of parameters $\theta$ using the conditional expectation of the complete-likelihood:

$$
\mathcal{Q}\left(\theta, \theta^{\prime}\right)=\mathbb{E}_{Y_{1: T}, \theta^{\prime}}\left[\log p_{\theta}\left(X_{0: T}, S_{0: T}, Y_{1: T}\right)\right] .
$$

Given an initial value $\theta^{\prime}$, the ML estimator is iteratively approached by $\theta$ which maximizes $\mathcal{Q}\left(\theta, \theta^{\prime}\right)$.

\subsubsection{Expectation Step}

Based on the interaction (6) between $X_{t}$ and $S_{t}$, the conditional expectation $\mathcal{Q}\left(\theta, \theta^{\prime}\right)$ can be written as follows

$$
\begin{aligned}
\mathcal{Q}\left(\theta, \theta^{\prime}\right)=\sum_{s_{0: T}}\left\{p_{\theta^{\prime}}\left(s_{0: T} \mid y_{1: T}\right)\right. \\
\left.\mathbb{E}_{S_{0: T}, Y_{1: T}, \theta^{\prime}}\left[\log p_{\theta}\left(X_{0: T}, S_{0: T}, Y_{1: T}\right)\right]\right\}
\end{aligned}
$$

Given $s_{0: T}$, the conditional expectation in (10) can be evaluated using a Kalman filter.

\subsubsection{Maximization Step}

Our aim is to maximize $\mathcal{Q}\left(\theta, \theta^{\prime}\right)$ w.r.t. $\theta$, under the trivial constraint on the transition matrix $\sum_{j} A(i, j)=1$. Then we derive the Lagrangian:

$$
\mathcal{L}(\theta, \lambda)=\mathcal{Q}\left(\theta, \theta^{\prime}\right)+\sum_{i=1}^{\kappa} \lambda_{i}\left[1-\sum_{j} A(i, j)\right],
$$

where $\lambda_{i}$ are the Lagrangian coefficients. Solving the derivative equations of $\mathcal{L}\left(\theta, \theta^{\prime}\right)$ w.r.t. $\theta$ leads to a system of $6 \cdot \kappa$ equations which require the calculation of $\tilde{x}_{t \mid T}=\mathbb{E}\left[x_{t} \mid\right.$ $\left.s_{0: T}, y_{1: T}, \theta\right]$ and $\tilde{x}_{t, r \mid T}=\mathbb{E}\left[x_{t} \cdot x_{r} \mid s_{0: T}, y_{1: T}, \theta\right]$ for all possible $s_{0: T} \in\{1, \ldots, \kappa\}^{T+1}$. However, an exact computation of these conditional expectations needs to perform summations over up to $\kappa^{T+1}$ values of $s_{0: T}$. Even for modest values of $T$, this requires a prohibitive computational cost.

\subsection{Monte Carlo approximation of the EM algorithm for SMSSM}

To overcome this problem, we resort to the particle filters method to numerically approximate the EM algorithm [9]. More precisely, we use a set of $N$ "particles" $\left\{s_{0: T}^{i}\right\}_{i=1}^{N}$ and importance weights $\left\{w_{T}^{i}\right\}_{i=1}^{N}$, such that $\partial \mathcal{Q}\left(\theta, \theta^{\prime}\right) / \partial \theta$ can be estimated by

$$
\sum_{i=1}^{N} w_{T}^{i} \frac{\partial}{\partial \theta} \mathbb{E}_{s_{0: T}^{i}, Y_{1: T}, \theta^{\prime}}\left[\log p_{\theta}\left(X_{0: T}, s_{0: T}^{i}, Y_{1: T}\right)\right] .
$$

The $N$ particle sequences can be sequentially simulated using the importance sampling [10]: starting from samples $s_{0: t-1}^{i}$, new samples $s_{t}^{i}$ are simulated according to an importance function $q_{\theta^{\prime}}\left(s_{t} \mid s_{0: t-1}, y_{1: t}\right)$. The associated importance weights satisfy $\sum_{i} w_{t}^{i}=1$, and can be calculated recursively according to the following formula

$$
w_{t}^{i} \propto w_{t-1}^{i} \frac{p_{\theta^{\prime}}\left(y_{t} \mid y_{1: t-1}, s_{1: t}\right) p_{\theta^{\prime}}\left(s_{t} \mid s_{1: t-1}\right)}{q_{\theta^{\prime}}\left(s_{t} \mid y_{1: t}, s_{1: t-1}\right)} .
$$

Here, we choose $q_{\theta^{\prime}}\left(s_{t} \mid y_{1: t}, s_{1: t-1}\right)=p_{\theta^{\prime}}\left(s_{t} \mid y_{1: t}, s_{1: t-1}\right)$ which minimizes the variance of the importance weights [10]. In practice after a few simulation iterations, a lot of importance weights could be very close to zero. To avoid this "degeneracy phenomenon", a selection step is generally introduced when the variance of the weights is higher than a predefined threshold: it consists of discarding the particles $s_{0: t}^{i}$ with low weights and duplicating the ones with high weights. It has to be noted that more adapted smoothing algorithms can be used to simulate the $N$ particles [11]. Algorithm 1 presents an iteration of the proposed Monte Carlo (MC) EM algorithm, with computational complexity equal to $O(N T)$.

The marginal likelihood $p_{\theta}\left(y_{1: T}\right)$ is approximated by:

$$
\hat{p}_{\theta}\left(y_{1: T}\right)=\hat{p}_{\theta}\left(y_{1}\right) \prod_{t=2}^{T} \hat{p}_{\theta}\left(y_{t} \mid y_{1: t-1}\right),
$$

where $\hat{p}_{\theta}\left(y_{t} \mid y_{1: t-1}\right)=\sum_{i=1}^{N} w_{t-1}^{i} p_{\theta}\left(y_{t} \mid s_{0: t-1}^{i}, y_{1: t-1}\right)$.

The next section describes among other the identification of the optimal number of Markov states as well as the online estimation of $\left(s_{t}, x_{t}\right)$ given an estimated $\theta$.

\section{NUMERICAL EXPERIMENTS}

To validate the new $S o C$ model, as well as the proposed method for parameters estimation, real-life Electric Vehicle 


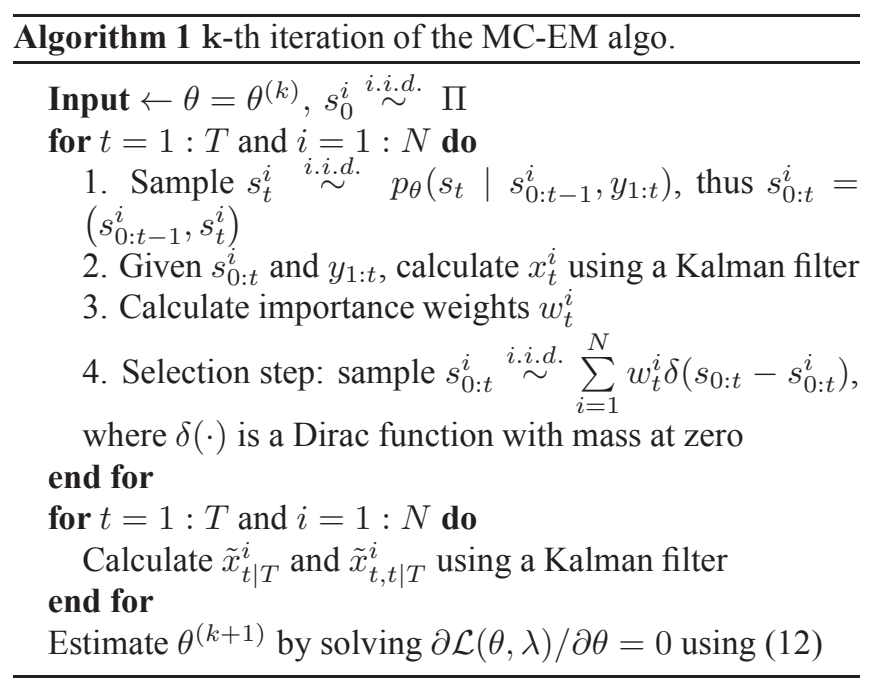

(EV) data is used. Accordingly, the battery usage does not only depend on ambient temperature and itinerary, but also on driver behavior and road conditions.

\subsection{Description of the learning dataset}

The learning dataset (Fig.2) comprises instantaneous current and voltage measurements collected during a drive of an $\mathrm{EV}$, with an ambient temperature equal to $15^{\circ} \mathrm{C}$, a sampling time of $2 s$ and a working time of $4500 \mathrm{~s}$. The $S o C$ was calculated using the Coulomb counting method as the EV was equipped with an accurate current sensor.

\subsection{Choice of the number of hidden Markov states}

The optimal number of hidden Markov states is identified by the trade-off between accuracy requirements and model complexity. Hence, seven $S o C$ models have been tested $(\kappa=$ $\{1, \ldots, 7\})$. For $\kappa=1$, the SMSSM is a simple linear statespace model. Thus two model selection criteria have been considered $[12,13]$ :
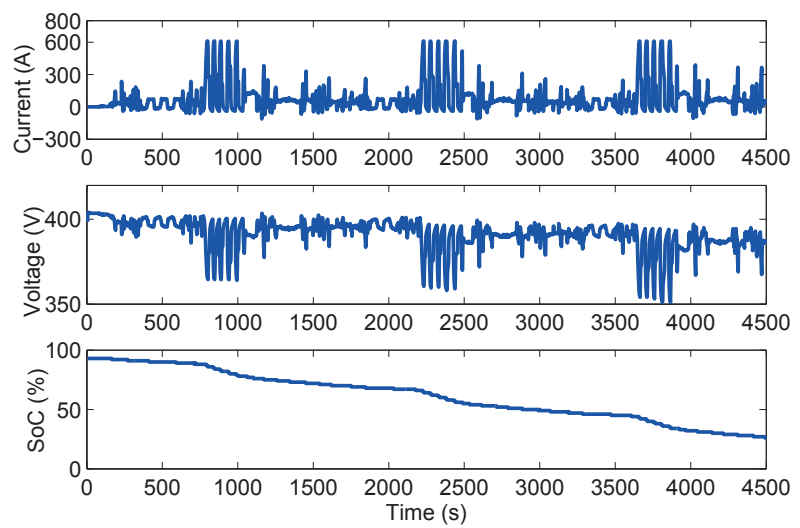

Fig. 2. Learning dataset collected during a drive of an EV

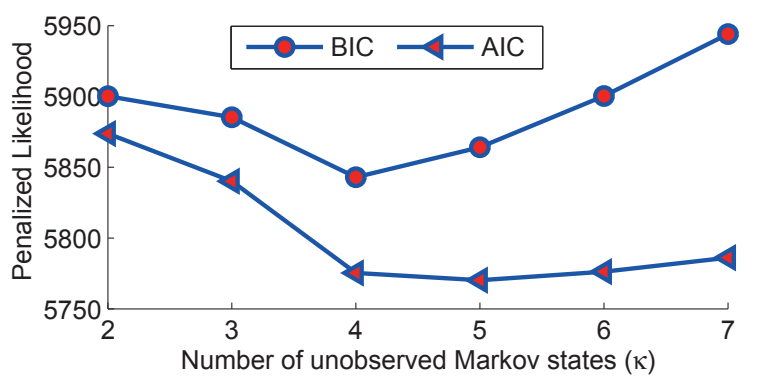

Fig. 3. Model selection criteria: $\operatorname{BIC}(\circ)$ and $\operatorname{AIC}(\triangle)$

$$
\begin{aligned}
& \mathrm{BIC}=-2 \log \left(\hat{p}_{\theta}\left(y_{1: T}\right)\right)+K \log (T) \\
& \mathrm{AIC}=-2 \log \left(\hat{p}_{\theta}\left(y_{1: T}\right)\right)+2 K,
\end{aligned}
$$

where $K=\kappa(\kappa+5)$ is the number of unknown parameters. As shown in Fig. 3, the model with $\kappa=4$ is considered as the "best" model according to BIC. However, AIC chooses $\kappa=5$ but hesitates between $\kappa=4,5$ and 6 . The results show that BIC leads to an easier interpretation of the model [14].

\subsection{Online estimation of the state of charge}

We suppose here that the number of hidden Markov states has been previously identified (see $\S 4.2$ ) and that the associated vector of parameters $\theta$ is estimated (see $\S 3.2$ ). Given a new observation $y_{t}$ and an input $u_{t}$, the $S o C$ is online estimated using a particle filter; i.e., $N$ particles $s_{t}^{i}$ are simulated from $p_{\theta}\left(s_{t} \mid s_{0: t-1}^{i}, y_{t}\right)$ (steps 1-4 of Algorithm 1), then $N x_{t}^{i}$ are obtained using the Kalman filter. Finally, $S o C_{t}$ is estimated by

$$
\hat{S o C_{t}}=\sum_{i=1}^{N} w_{t}^{i} \cdot x_{t}^{i},
$$

and confidence interval can be constructed.

\subsection{Validation of the model}

The learned SMSSM under $15^{\circ} C$ with $\kappa=4$ is tested using three different datasets also collected during a drive of an EV, under different ambient temperatures $\left(5,15,25^{\circ} \mathrm{C}\right)$. The results show that SMSSM provides an accurate $S o C$ estimation even with ambient temperatures different than that of the learning dataset. Indeed, the maximum difference between the $S o C$ estimated by Coulomb counting and SMSSM is equal to $5 \%$; whereas this difference reaches $20 \%$ for a single linear state-space model, Fig. 4. Moreover, numerical experiments show that under a specific hidden Markov state, the relation between $S o C$, voltage and current is linear (Fig.5) which confirms our starting hypothesis ( $\$ 2.2)$. Figure 6 shows that the hidden Markov state might reflect a specific usage of the battery as it follows closely the variation of the voltage. Thus, we expect that this hidden state would have a physical interpretation and would model the physical changes of the battery behavior. 


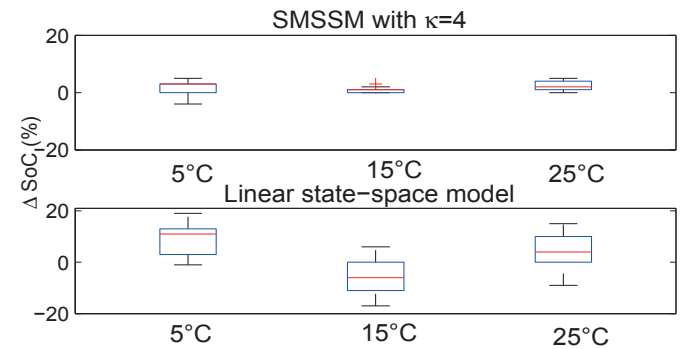

Fig. 4. Boxplots of $\triangle S o C=S o C$ (Ah-counting) $S o C$ (SMSSM) with $\kappa=4$ (top) and Linear state-space model (bottom) for ambient temperature equal to $5,15,25^{\circ} \mathrm{C}$
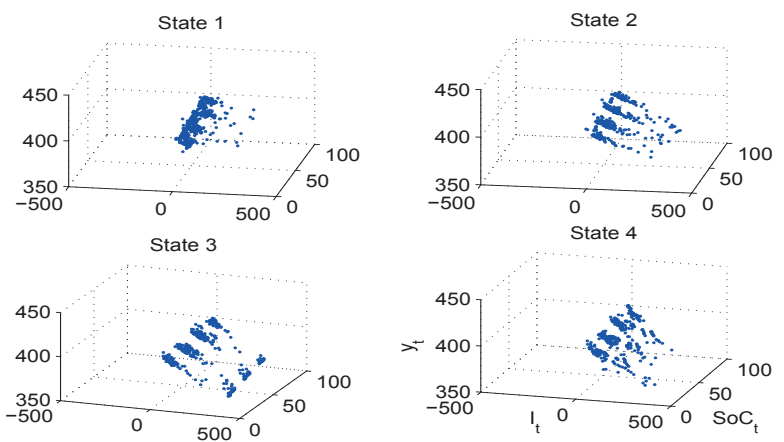

Fig. 5. Voltage vs $S o C$ vs current: instantaneous measurements under each hidden Markov state for a SMSSM with $\kappa=4$

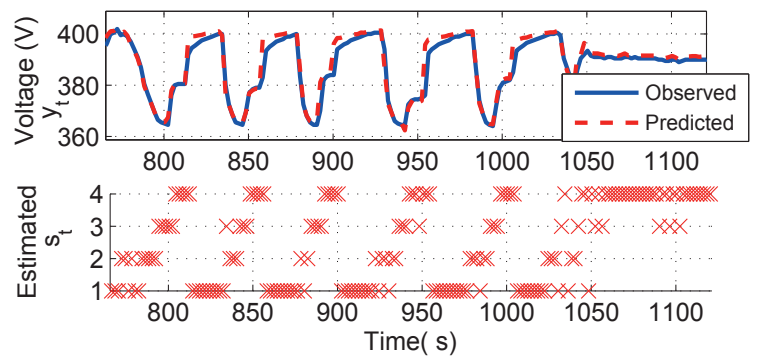

Fig. 6. Variation of the voltage and the hidden Markov state over time. The presented $s_{t}$ is the one that appears the most in the $N$ particles $s_{t}^{i}$

\section{CONCLUSION}

In this paper, we have proposed a new model for $S o C$ estimation. This model relates to Switching Markov State-Space Models which are a linear state-space model with parameters indexed by a Markov chain. The unknown parameters are estimated using a MC approximation of the EM algorithm. Validation on an EV real data confirmed the ability of the proposed model to accurately estimate the $S o C$ for different drives and ambient temperatures. In addition, the results show that the hidden Markov state might reflect a specific usage of the battery. Future work needs to focus on its phys- ical interpretation based on the internal and external usage conditions. Moreover, a comparison between our MC-EM algorithm and a full Bayesian approach, specifically the Gibbs sampling, still need to be explored.

\section{REFERENCES}

[1] J. Kalawoun, K. Biletska, F. Suard, and M. Montaru, "From a novel classification of the battery state of charge estimators toward a conception of an ideal one," Journal of Power Sources, vol. 279, pp. 694-706, 2015.

[2] T. Hirai, A. Ohnishi, N. Nagaoka, N. Mori, A. Ametani, and S. Umeda, "Automatic equivalent-circuit estimation system for lithium-ion battery," Universities Power Engineering Conference, pp. 1-5, Sept 2008.

[3] S. Pang, J. Farrell, J. Du, and M. Barth, "Battery stateof-charge estimation," American Control Conference, vol. 2, pp. 1644-1649, 2001.

[4] G. Plett, "Extended Kalman filtering for battery management systems of LiPB-based HEV battery packs," Journal of Power Sources, vol. 134, pp. 262-276, 2004.

[5] K. S. Ng, C. S. Moo, Y. P. Chen, and Y. C. Hsieh, "Enhanced Coulomb counting method for estimating stateof-charge and state-of-health of lithium-ion batteries," Applied energy, vol. 86, no. 9, pp. 1506-1511, 2009.

[6] D. O. Anderson and J. B. Moore, Optimal filtering, Englewood Clifss, N. J. Prentice-Hall, 1979.

[7] S. Frühwirth-Schnatter, Finite Mixture and Markov Switching Models, Springer, 2006.

[8] A. P. Dempster, N. M. Laird, and D. B. Rubin, "Maximum likelihood from incomplete data via the EM algorithm," Journal of the Royal Statistical Society. Series $B$, vol. 39, pp. 1-38, 1977.

[9] Martin A. Tanner, Tools for Statistical Inference - Observed Data and Data Augmentation Methods, vol. 67, Springer-Verlag New York, 1991.

[10] A. Doucet and A. M. Johansen, "A tutorial on particle filtering and smoothing: Fifteen years later," Handbook of Nonlinear Filtering, vol. 12, pp. 656-704, 2009.

[11] P. Fearnhead, D. Wyncoll, and J. Tawn, "A sequential smoothing algorithm with linear computational cost," Biometrika, vol. 97, no. 2, pp. 447-464, 2010.

[12] H. Akaike, "A new look at the statistical model identification," IEEE Transactions on Automatic Control, vol. 19, no. 6, pp. 716-723, Dec 1974.

[13] G. Schwarz, "Estimating the Dimension of a Model," The Annals of Statistics, vol. 6, no. 2, pp. 461-464, Mar 1978.

[14] K.P. Burnham and D.R. Anderson, Model selection and multimodel inference: a practical information-theoretic approach, Springer, 2002. 